\title{
Importance of magnetic resonance imaging in the diagnosis and prognosis of apical hypertrophic cardiomyopathy: Case report and literature review
}

\author{
Importancia de la resonancia magnética en el diagnóstico y pronóstico de la \\ cardiomiopatía hipertrófica apical, a propósito de un caso clínico
}

\author{
Raul Alca-Clares ${ }^{1}$, Jorge Salinas-Arce ${ }^{2 *}$, Henry Anchante-Hernández ${ }^{1}$, and Félix Medina-Palomino ${ }^{1}$ \\ ${ }^{1}$ Department of Cardiology, Hospital Cayetano Heredia; ${ }^{2}$ Arrhythmia Unit, Clínica Delgado, Lima, Peru
}

Hypertrophic cardiomyopathy (HCM) is the most common inherited heart disease and the second cause of sudden cardiac death (SCD) after coronary artery disease ${ }^{1}$.

One of the rarest variants is apical HCM (AHCM). AHCM risk stratification and cardioverter defibrillator (CD) implantation as primary prevention are a complex decision, which in most cases is not included in European and American stratification models.

We present the case of a 41 -year-old female patient, with no cardiovascular history and undergoing pre-surgical evaluation for cholecystectomy. She did not refer palpitations or syncope.

On electrocardiogram, she showed normal sinus rhythm, QRS complexes of increased amplitude, and diffuse repolarization changes (Fig. 1A).

On transthoracic echocardiography, the left ventricular (LV) maximum wall thickness was observed at the apical level $(21 \mathrm{~mm})$, with extension to the mid-ventricular third (Fig. 1B and C). LV outflow tract gradient was estimated at $8 \mathrm{mmHg}$ and mid-ventricular gradient was $33 \mathrm{mmHg}$ (Fig. 1D). Global longitudinal strain (GLS) was $-12 \%$ (Fig. 1E).
According to the European stratification model, estimated SCD risk for the following 5 years was $2.06 \%$ (low risk); according to the American model, no conventional risk factors were identified. Both tools did not recommend CD implantation as primary prevention.

The magnetic resonance imaging evaluation found apical akinesia and ventricular cavity obliteration during systole (Fig. 2A and B), with late gadolinium enhancement (LGE) in the areas of highest hypertrophy, quantified at $23.9 \%$ (Fig. 2C).

The myocardial perfusion study did not show inducible ischemia. The apical region showed higher brightness of the tracer (Fig. 2D).

Considering that in our case, clinical guidelines are insufficient for risk stratification, and based on the presence of AHCM with apical aneurysm (AA), extensive LGE, mid-ventricular obstruction, and GLS alteration, we recommended CD implantation and anticoagulation initiation.

AHCM constitutes $4 \%$ of the different HCM phenotypes and has a cardiac mortality of $0.5 \% /$ year $^{2}$. However, no risk stratification model individualizes this type of patients. license (http://creativecommons.org/licenses/by-nc-nd/4.0/).
Available online: 25-05-2021 Arch Cardiol Mex (Eng). 2021;91(1):132-135 www.archivoscardiologia.com 


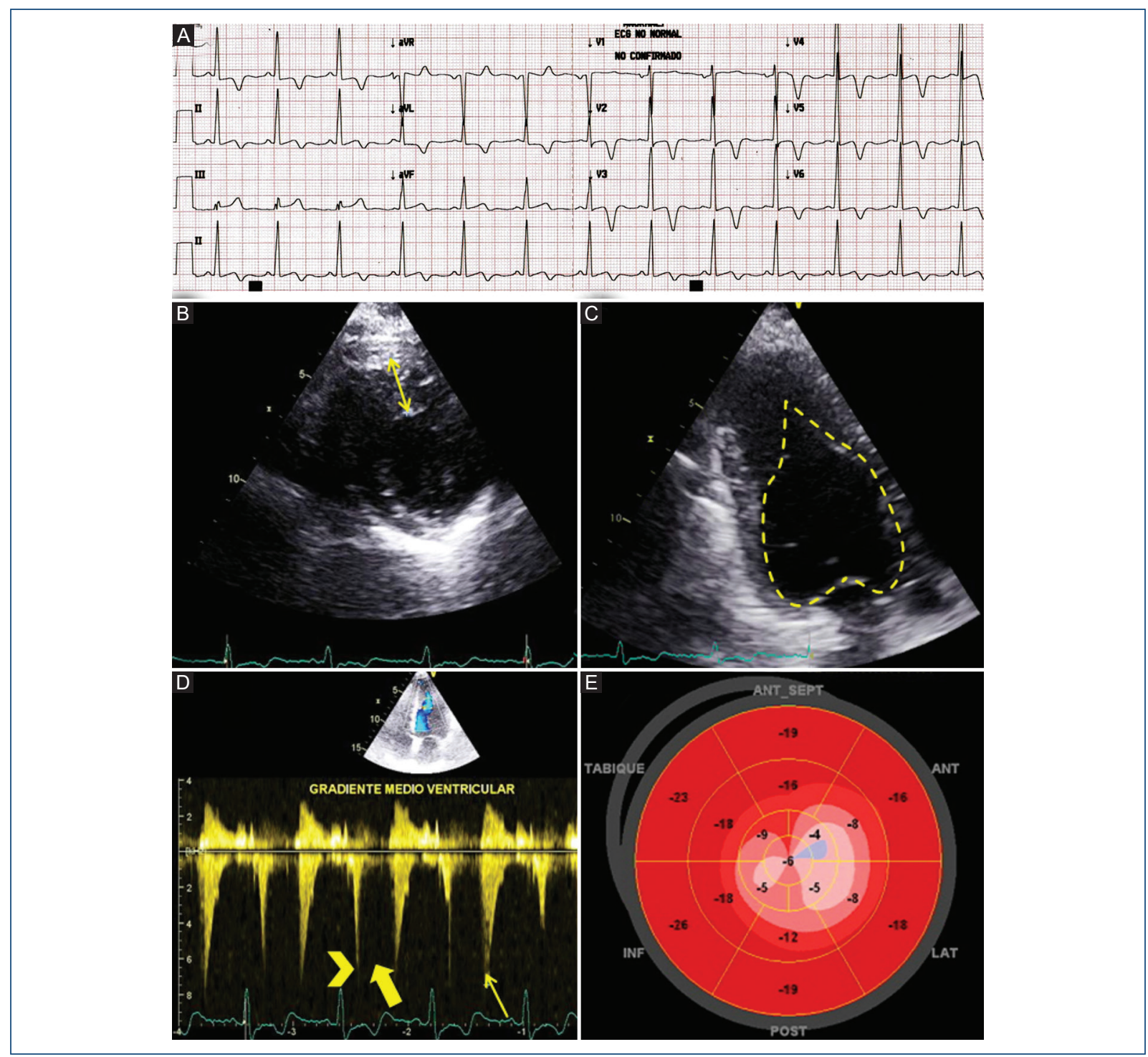

Figure 1. A: electrocardiogram. Deep $S$ wave in $\mathrm{V} 1(13 \mathrm{~mm})$ and high $\mathrm{R}$ wave in $\mathrm{V} 5(28 \mathrm{~mm})$; negative T waves $(-8 \mathrm{~mm}$ in V3) from V2 through V6, DI and aVL; absence of 0 waves in lateral leads; J point elevation $(1 \mathrm{~mm})$ in DIII and aVF, with $r R^{\prime}$ pattern in DIII and R wave slurring in aVF. Echocardiography. B: left parasternal short axis at the apical level, maximum end-diastolic thickness of $21 \mathrm{~mm}$. C: 3 -chamber apical view, showing the ventricular cavity in the shape of an "ace of spades" at end-diastole and apical and mid-ventricular thickening. D: mid-ventricular turbulence with maximum gradient indicated by the arrowhead, followed by a Doppler signal void at mid-systole (thick arrow) and a paradoxical jet flow at protodiastole (thin arrow). E: bull's eye image. Predominantly apical segmental alteration.

Association with AA, observed in up to $20 \%$ of cases, and presence of an apical phenotype with mid-ventricular extension (type B AHCM), as observed in our patient, is associated with a 3-fold higher risk of SCD and greater probability of progression to heart failure ${ }^{3,4}$.

$\mathrm{HCM}$ evaluation by magnetic resonance imaging and LGE quantification is of special value in the subgroup of patients defined as "low risk" (without conventional risk factors). LGE is significantly correlated with SCD events, a LGE increase $>15 \%$ is associated with a risk even higher than $6 \% / 5$ years $^{5}$. In particular, patients with $\mathrm{AHCM}$ and $\mathrm{AA}$ show a significant risk increase even with the presence of LGE $>5 \%{ }^{6}$.

The inclusion of LGE and the presence of $A A$ as independent variables prospectively identified $95 \%$ of patients who developed life-threatening arrhythmias, preventing almost all cases of $\mathrm{SCD}^{7}$. At present, magnetic resonance imaging not only plays a role in the identification of the different morphological patterns of $\mathrm{HCM}$ but is also a useful tool in risk stratification for 


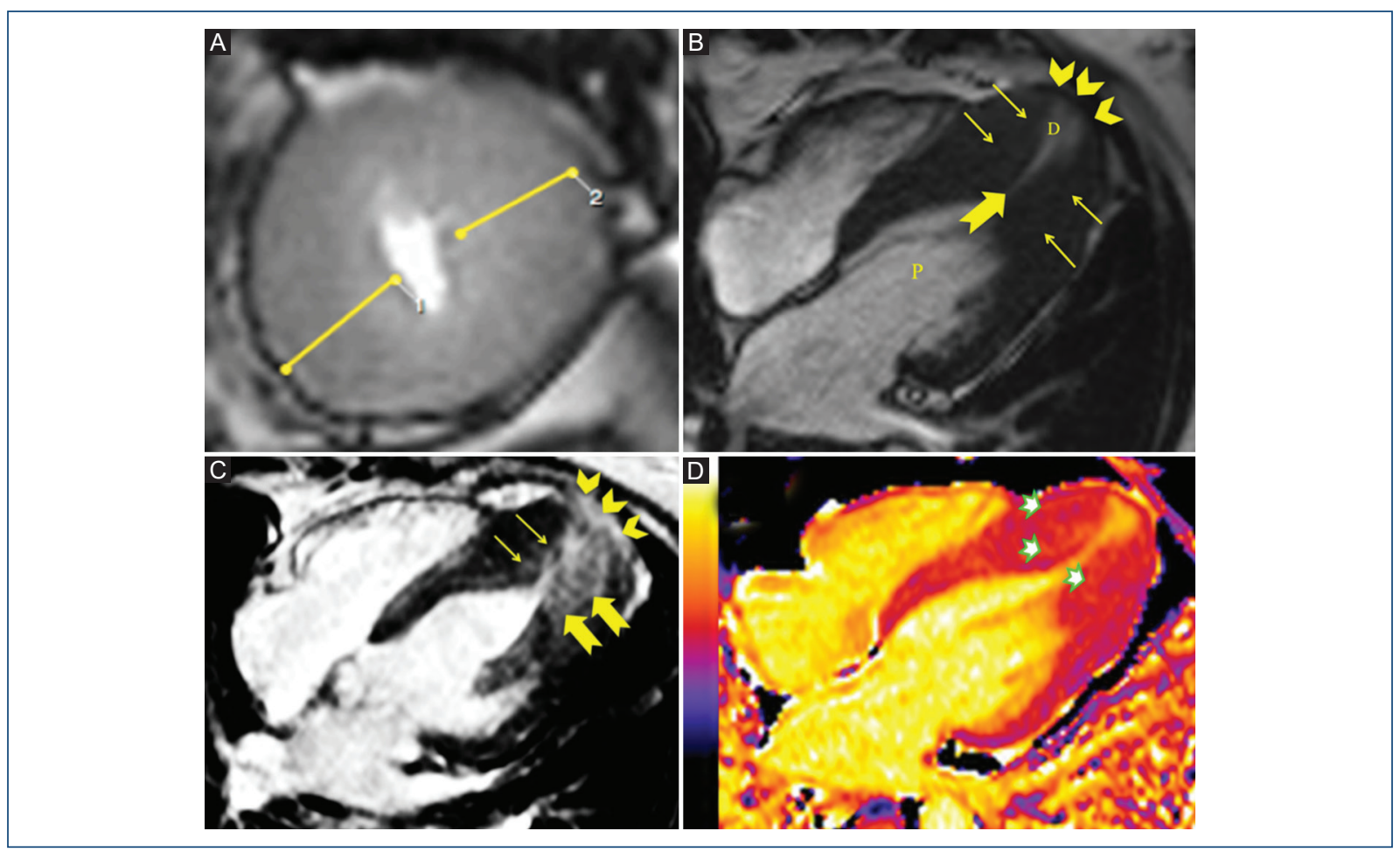

Figure 2. Cardiac magnetic resonance imaging. A: apical short axis, maximum wall thickness at 23 mm. B: four-chamber view. Seventeen millimeter apical aneurysm (arrowhead), with no presence of thrombus. Apical hypertrophy spreads to the papillary muscles' base (thin arrows) with mid-ventricular obstruction due to anterolateral and septal wall coaptation during systole (thick arrow), which generates a proximal (P) and another distal (D) cavity. C: late gadolinium enhancement (LGE). A non-ischemic patchy LGE pattern is observed at the apex and aneurysmal border (arrowhead) with extension to contiguous areas of the anterolateral wall distal (thick arrows) and inferoseptal portions (thin arrows). D: T1 mapping. Signal increase up to values of 1.077-1.104 ms in areas of maximum hypertrophy. Normality ranges were between 960 and $1060 \mathrm{~ms}$.

sudden death, especially in those patients defined as "low risk" according to stratification models ${ }^{8}$.

Risk stratification in patients with AHCM for cardiovascular complications must be carried out individually, as they constitute a group with special characteristics that include risk factors not yet recognized in current guidelines.

\section{Funding}

This research has not received any specific grant from agencies in the public, commercial, or non-profit sectors.

\section{Conflicts of interest}

The authors declare that they have no conflicts of interest.

\section{Ethical disclosures}

Protection of human and animal subjects. The authors declare that no experiments were performed on humans or animals for this research.

Confidentiality of data. The authors declare that they have followed the protocols of their work center on the publication of patient data.

Right to privacy and informed consent. The authors declare that no patient data appear in this article.

\section{References}

1. Weissler-Snir A, Allan K, Cunningham K, Connelly KA, Lee DS, Spears DA, et al. Hypertrophic cardiomyopathy-related sudden cardiac death in young people in Ontario. Circulation. 2019;140(21):1706-16.

2. Klarich KW, Attenhofer Jost $\mathrm{CH}$, Binder J, Connolly HM, Scott CG, Freeman WK, et al. Risk of death in long-term follow-up of patients with apical hypertrophic cardiomyopathy. Am J Cardiol. 2013;111(12): 1784-91. 
3. Kubo T, Kitaoka H, Okawa M, Hirota T, Hoshikawa E, Hayato K, et al. Clinical profiles of hypertrophic cardiomyopathy with apical phenotypecomparison of pure-apical form and distal-dominant form. Circ $\mathrm{J}$ Off $\mathrm{J}$ Jpn Circ Soc. 2009;73(12):2330-6.

4. Yan L-R, Zhao S-H, Wang H-Y, Duan F-J, Wang Z-M, Yang Y-J, et al. Clinical characteristics and prognosis of 60 patients with midventricular obstructive hypertrophic cardiomyopathy. J Cardiovasc Med Hagerstown Md. 2015:16(11):751-60.

5. Chan RH, Maron BJ, Olivotto I, Pencina MJ, Assenza GE, Haas T, et al. Prognostic value of quantitative contrast-enhanced cardiovascular magnetic resonance for the evaluation of sudden death risk in patients with hypertrophic cardiomyopathy. Circulation. 2014;130(6):484-95.
6. Hanneman K, Crean AM, Williams L, Moshonov H, James S, Jiménez-Juan $L$, et al. Cardiac magnetic resonance imaging findings predict major adverse events in apical hypertrophic cardiomyopathy. J Thorac Imaging. 2014;29(6):331-9.

7. Maron MS, Rowin EJ, Wessler BS, Mooney PJ, Fatima A, Patel P, et al. Enhanced American College of Cardiology/American Heart Association Strategy for Prevention of Sudden Cardiac Death in High-Risk Patients With Hypertrophic Cardiomyopathy. JAMA Cardiol. 2019;4(7):644-57.

8. Todiere G, Nugara C, Gentile G, Negri F, Bianco F, Falletta C, et al. Prognostic role of late gadolinium enhancement in patients with hypertrophic cardiomyopathy and low-to-intermediate sudden cardiac death risk score. Am J Cardiol. 2019;124(8):1286-92. 